\title{
Theoretical Investigation on the Contact Duration in Elastic and Inelastic Collision of a Circular Plate to a Planar Surface*
}

\begin{abstract}
Zhongjie LU* ${ }^{* *}$ Koichi TANAKA ${ }^{* * *}$, Masahiro NISHIDA ${ }^{* * *}$ and Fenglei HUANG****
In this paper a new theoretical method is proposed for calculating the normalized contact duration and indentation depth in elastic, elastic-plastic and plastic collision of circular plates. The contact duration is composed of total loading time and unloading time that are dependent on the relationship between the interaction force and normal indentation. For plastic collision, the contact duration relates to the coefficient of restitution (COR) of the collision. Considering the actual thickness of a circular plate, a coefficient of indentation (COI) is newly introduced in theoretical analyses of the COR. According to experimental results of COR, a hybrid nonlinear model is also proposed to describe the COR of circular plates for obtaining the unloading time of the plastic collision. Contact force history during collision can be expressed approximately by a half-sine wave and the force and contact duration is calculated numerically.
\end{abstract}

Key Words: Contact Problem, Contact Duration, Coefficient of Restitution, Plastic Working, Inelasticity, Circular Plate

\section{Introduction}

The study of contact mechanics is an important branch of the field relating to dynamic behavior of solid bodies. Conventional contact problems of two solid bodies in a quasi-static state are based on Hertz contact theory $^{(1)}$. When bodies collide, they approach with a relative velocity at an initial point of contact. The normal component of contact force would result in the deformation near the contact point and increases as the deformable body is compressed. The normal component of the relative velocity vanishes when the indentation in the normal direc-

* Received 17th June, 2005 (No. 05-4080)

** Department of Engineering, Physics, Electronics and Mechanics, Graduate Course, Nagoya Institute of Technology, Gokiso-cho, Showa-ku, Nagoya 466-8555, Japan. E-mail: lvzhongjie@hotmail.com

*** Department of Engineering, Physics, Electronics and Mechanics, Graduate School, Nagoya Institute of Technology, Gokiso-cho, Showa-ku, Nagoya 466-8555, Japan. E-mail: tanaka.koichi@nitech.ac.jp; nishida.masahiro@nitech.ac.jp

**** State Key Lab. of Explosion Science and Technology, Beijing Institute of Technology, 5 South Street of Zhongguanchun, District of Haidian, Beijing 100081, P.R. China. E-mail: huangfl@bit.edu.cn tion reaches it's maximum and the elastic strain energy is stored up to a maximum level. The elastic strain energy generates a force that drives the bodies apart in a relative restitution velocity.

A collision between hard bodies occurs in a very short period, which has been used to justify the rigid body impact theory in which the bodies instantaneously change the velocity when they collide. Although a contact duration is not an intrinsic material property, it is a global measure of the characteristic of the impact. For elastic spheres, the contact duration has been calculated ${ }^{(2)}$, which is proportional to the radius of the spheres and inversely proportional to the approach velocity. Landau, L.D. ${ }^{(3)}$ has also introduced the deduction of contact duration in studying the frictionless elastic contact. In the range of plastic deformation, the amount of elastic strain energy is different from that in the state of the elastic and the contact duration cannot be considered as a simple algebraic relation. Therefore, the difference in the contact duration can be used to illustrate the energy-loss in inelastic collisions.

Contact duration is closely related to coefficient of restitution (COR) that relates to the approach velocity. Among many hypotheses, a hybrid nonlinear collision model $^{(4)}$ for COR gives good agreement with experimental results ${ }^{(5)}$

Although some numerical simulations about the pla- 
nar (2D) collision of rough elastic-plastic circular plate have been made ${ }^{(6)-(8)}$, minute discussions on a contact duration of collision for elastic, elastic-plastic and full plastic circular plate have not been yet mentioned. When two bodies of rate-independent materials collide, the indentation occurs simultaneously with the interaction force. For circular plate, the relationship between the interaction force and the normal indentation is different from that in spheres $^{(9)}$. In this study, the contact duration of circular plate is derived from the plastic work and kinetic energy.

\section{Elastic Collision}

The elastic compression of two-dimensional bodies in contact cannot be calculated solely from the contact stress given by Hertz theory. For the contact of long cylindrical bodies, the ends of the cylinder are assumed to be in the state of plane stress. When an infinitely long cylinder with the radius $R$ impacts to a plane, the normal indentation, $\delta$ is given by ${ }^{(9)}$,

$$
\delta=\frac{b^{2}}{2 R}\left[2 \ln \left(\frac{4 R}{b}\right)-1\right]
$$

where normal indentation of the circular plate $\delta$ is shown in Fig. 1.

According to Hertz theory, the relationship of half width of the contact surface, $b$, and the compressive load per unit axial length, $P$, is given by

$$
b^{2}=4 P R / \pi E_{*}
$$

where $E_{*}$ is the composite elastic modulus of the cylinder and plane.

$$
\frac{1}{E_{*}}=\frac{1-v_{1}^{2}}{E_{1}}+\frac{1-v_{2}^{2}}{E_{2}}
$$

where $E_{1}, v_{1}, E_{2}, v_{2}$ are Young's modulus and Poisson's ratio of the cylinder and the plane, respectively.

Substituting Eq. (2) into Eq. (1), we obtain

$$
\delta=\frac{2 P}{\pi E_{*}}\left[\ln \left(\frac{4 R \pi E_{*}}{P}\right)-1\right]
$$

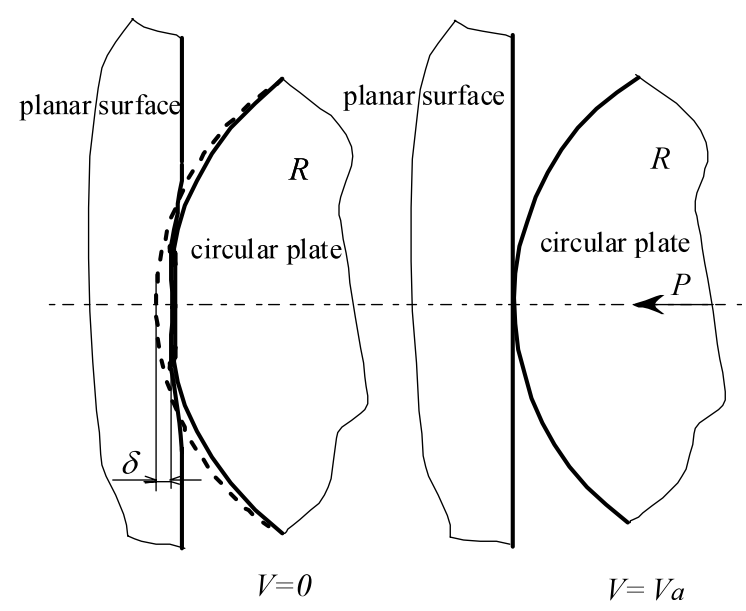

Fig. 1 Compression and indentation of circular plate and planar surface
Equation (4) is transformed into a differential form as

$$
\frac{d \delta}{d P}=\frac{2}{\pi E_{*}}\left[\ln \left(\frac{4 R \pi E_{*}}{P}\right)-2\right]
$$

If it is assumed that all of the strain energy in a deformed body is caused by the change of kinetic energy, we can write

$$
\begin{aligned}
& \frac{1}{2} m V_{a}^{2}-\frac{1}{2} m(\dot{\delta})^{2}=\int P d \delta \\
& =\int \frac{2 P}{\pi E_{*}}\left[\ln \left(\frac{4 R \pi E_{*}}{P}\right)-2\right] d P
\end{aligned}
$$

where $V_{a}$ is the approach velocity of the cylinder at the beginning of impact. We obtain

$$
V_{a}^{2}-(\dot{\delta})^{2}=\frac{2 P^{2}}{m \pi E_{*}}\left[\ln \left(\frac{4 R \pi E_{*}}{P}\right)-\frac{3}{2}\right]
$$

Since the maximum compressive load, $P_{m}$, acts when $\dot{\delta}$ vanishes, we have

$$
V_{a}^{2}=\frac{2 P_{m}^{2}}{m \pi E_{*}}\left[\ln \left(\frac{4 R \pi E_{*}}{P_{m}}\right)-\frac{3}{2}\right]
$$

and

$$
\delta=\frac{m V_{a}^{2}}{P_{m}}+\frac{P_{m}}{\pi E_{*}}
$$

For calculating the contact duration, Eq. (7) is written as

$$
\left(\frac{d \delta}{d P} \cdot \frac{d P}{d t}\right)^{2}=V_{a}^{2}-\frac{2 P^{2}}{m \pi E_{*}}\left[\ln \left(\frac{4 R \pi E_{*}}{P}\right)-\frac{3}{2}\right]
$$

Using Eq. (5) in Eq. (10), we have

$$
d t=\frac{\frac{2}{\pi E_{*}}\left[\ln \left(\frac{4 R \pi E_{*}}{P}\right)-2\right]}{\sqrt{V_{a}^{2}-\frac{2}{m \pi E_{*}}\left[\ln \left(\frac{4 R \pi E_{*}}{P}\right)-\frac{3}{2}\right] P^{2}}} d P
$$

Putting $P / P_{m}=x$ and using Eq. (8), the elastic contact duration, $t_{c e}$, is given by

$$
t_{c e}=\int_{0}^{1} \frac{\frac{2}{\pi E_{*}}\left[\ln \left(\frac{4 R \pi E_{*}}{P_{m}}\right)-\ln x-2\right] P_{m} d x}{\sqrt{V_{a}^{2}\left(1-x^{2}\right)+\frac{2 P_{m}^{2}}{m \pi E_{*}} x^{2} \ln x}}
$$

For the elastic collision, the contact duration is a sum of two parts: the period of elastic loading, $t_{e l}$, and the period of elastic unloading, $t_{\text {eunl }}$. Since the COR of the elastic collision is equal to 1 , the contact duration is the twice of the integrated time given by Eq. (12). A Romberg method of numerical integration can be used to compute the numerical value of Eq. (12).

The normalized loading time, contact duration and indentation depth as a function of the normalized approach velocity are shown in Fig. 2. $V_{a}, t_{c}$, and $\delta$ are the approach velocity, contact duration, indentation depth, respectively. $V_{Y}$ is the impact velocity that causes the yield of material. $\delta_{Y}$ is the maximum indentation depth at the yield velocity. $t_{Y}$ is the time to require the maximum indentation depth. 


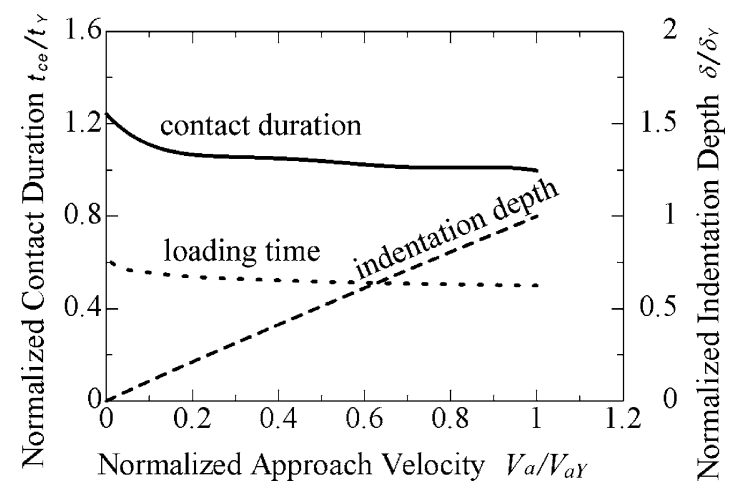

Fig. 2 Normalized contact duration, loading time or unloading time, normalized indentation depth as a function of normalized approach velocity

Figure 1 illustrates that the normalized contact duration slowly decreases with increasing approach velocity. At the same time, the indentation depth increases continuously and arrives at a maximum value of 1 when the approach velocity becomes the yield velocity.

Moreover, as the approach velocity is infinitesimally small, the compressive load would be small and the normalized contact duration would become infinite.

\section{Elastic-Plastic Collision}

The elastic-plastic deformation is initiated when the mean contact pressure equals to $1.5 Y$, where $Y$ is the yield stress of relevant material, this is called Von-Mises criterium by Johnson ${ }^{(9)}$. As a result of impact, the structural component of specimen undergoes local plastic deformation. An elastic subsurface layer would recover to their original shape during unloading. However, a compressive residual stress field is developed in the near-surface layer of the structural component.

The yield indentation, yield approach velocity, yield interaction force and yield loading time are denoted as $\delta_{Y}$, $V_{Y}, P_{Y}$ and $t_{Y}$, respectively when the elastic-plastic deformation is initiated. The elastic-plastic deformation would be terminated when the mean contact pressure equals to $2.4 Y^{(4)}$. At that time, the indentation, approach velocity, interaction force and loading time are denoted as $\delta_{P Y}$, $V_{P Y}, P_{P Y}$ and $t_{P Y}$. It is suggested that the total time of the elastic-plastic collision, $t_{\text {eptotal }}$, is divided into three parts: the period of elastic-plastic loading, $t_{e p l}$, the period of elastic loading, $t_{Y}$, and the period of elastic unloading, $t_{\text {epunl }}$.

$$
t_{\text {eptotal }}=t_{Y}+t_{\text {epl }}+t_{\text {epunl }}
$$

The relationship between the interaction force and normal indentation for quasi-static elastic-plastic collision has been suggested by Stronge ${ }^{(4)}$.

$$
\begin{aligned}
\frac{P}{P_{Y}} & =\left[1+\frac{R \delta_{Y}}{b_{Y}^{2}}\left(\frac{\delta}{\delta_{Y}}-1\right)\right]^{1 / 2} \\
& \times\left\{1+\frac{\sqrt{3}}{9} \ln \left[1+\frac{R \delta_{Y}}{b_{Y}^{2}}\left(\frac{\delta}{\delta_{Y}}-1\right)\right]\right\}
\end{aligned}
$$

where the yield interaction force, $P_{Y}$, and the yield half width of the contact surface, $b_{Y}$, are denoted respectively by

$$
\frac{P_{Y}}{Y R}=\frac{36}{\pi} \frac{Y}{E_{*}} \quad \frac{b_{Y}}{R}=\frac{12}{\pi} \frac{Y}{E_{*}}
$$

From Eq. (14), we have

$$
\begin{aligned}
P & =\frac{36 Y^{2} R}{\pi E_{*}}\left[1+\frac{R \delta_{Y}}{b_{Y}^{2}}\left(\frac{\delta}{\delta_{Y}}-1\right)\right]^{1 / 2} \\
& \times\left\{1+\frac{\sqrt{3}}{9} \ln \left[1+\frac{R \delta_{Y}}{b_{Y}^{2}}\left(\frac{\delta}{\delta_{Y}}-1\right)\right]\right\} \\
\frac{1}{2} & m V_{a}^{2}-\frac{1}{2} m(\dot{\delta})^{2}=\frac{36 Y^{2} R}{\pi E_{*}} \int_{\delta_{Y}}^{\delta}\left[1+\frac{R \delta_{Y}}{b_{Y}^{2}}\left(\frac{\delta}{\delta_{Y}}-1\right)\right]^{1 / 2} \\
& \times\left\{1+\frac{\sqrt{3}}{9} \ln \left[1+\frac{R \delta_{Y}}{b_{Y}^{2}}\left(\frac{\delta}{\delta_{Y}}-1\right)\right]\right\} d \delta \\
\delta_{Y} & =\frac{72 R Y^{2}}{\pi E_{*}^{2}}\left[\ln \left(\frac{\pi E_{*}^{2}}{9 Y^{2}}\right)-1\right]
\end{aligned}
$$

and

$$
\begin{aligned}
& V_{a}^{2}-(\dot{\delta})^{2}=\frac{48 Y^{2} b_{Y}^{2}}{m \pi E_{*}} \\
& \quad \times\left[A_{\delta}^{3 / 2}\left(1+\frac{\sqrt{3}}{9} \ln A_{\delta}-\frac{2 \sqrt{3} b_{Y}^{2}}{27 R}\right)+\frac{2 \sqrt{3} b_{Y}^{2}}{27 R}-1\right]
\end{aligned}
$$

where $A_{\delta}=1-\frac{R}{b_{Y}^{2}} \delta_{Y}+\frac{R}{b_{Y}^{2}} \delta$

Since the maximum indentation, $\delta_{m}$, acts when the approach velocity is $V_{a}$, we have

$$
\begin{aligned}
V_{a}^{2} & =V_{Y}^{2}+\frac{48 Y^{2} b_{Y}^{2}}{m \pi E_{*}} \\
& \times\left[A_{\delta_{m}}^{3 / 2}\left(1+\frac{\sqrt{3}}{9} \ln A_{\delta_{m}}-\frac{2 \sqrt{3} b_{Y}^{2}}{27 R}\right)+\frac{2 \sqrt{3} b_{Y}^{2}}{27 R}-1\right]
\end{aligned}
$$

where $\quad A_{\delta_{m}}=1-\frac{R}{b_{Y}^{2}} \delta_{Y}+\frac{R}{b_{Y}^{2}} \delta_{m}$

Using Eq. (19), the differential equation of loading time for the elastic-plastic loading is given by

$$
d t=\frac{d \delta}{\sqrt{V_{a}^{2}-\frac{48 Y^{2} b_{Y}^{2}}{m \pi E_{*}}\left[A_{\delta}^{3 / 2}\left(1+\frac{\sqrt{3}}{9} \ln A_{\delta}-\frac{2 \sqrt{3} b_{Y}^{2}}{27 R}\right)+\frac{2 \sqrt{3} b_{Y}^{2}}{27 R}-1\right]}}
$$

Putting $\delta / \delta_{m}=x$ and using Eq. (21), we find 


$$
t=\int_{\delta / \delta_{m}}^{1} \frac{\delta_{m} d x}{\sqrt{V_{Y}^{2}+\frac{48 Y^{2} b_{Y}^{2}}{m \pi E_{*}}\left[A_{\delta_{m}}^{3 / 2}\left(1+\frac{\sqrt{3}}{9} \ln A_{\delta_{m}}-\frac{2 \sqrt{3} b_{Y}^{2}}{27 R}\right)-A_{\delta_{m} x}^{3 / 2}\left(1+\frac{\sqrt{3}}{9} \ln A_{\delta_{m} x}-\frac{2 \sqrt{3} b_{Y}^{2}}{27 R}\right)\right]}}
$$

where $\quad A_{\delta_{m} x}=1-\frac{R}{b_{Y}^{2}} \delta_{Y}+\frac{R}{b_{Y}^{2}} \delta_{m} x$

The elastic-plastic loading time in Eq. (24) can be integrated numerically by Romberg method. Figure 3 shows the normalized loading time and the indentation depth as a function of the normalized approach velocity of a circular plate due to the elastic-plastic loading. As the normalized approach velocity increases the normalized indentation depth and normalized loading time increase. However, the phase of elastic-plastic deformation is in a very short period as shown in the Fig. 3. The elastic-plastic normalized approach velocity region is barely one-thousandth of the yield approach velocity.

The elastic-plastic total loading time is composed of two parts while the approach velocity is in the range of elastic-plastic velocity. The first part stays in the elasticplastic loading time. The second part is the elastic loading time, which is represented in section 2.

Since the unloading time does not change as the approach velocity increase, as shown in Fig. 4, the elasticplastic velocity is almost same as the yield velocity. The coefficient of restitution may be approximated by unity. The rebound velocity can be considered as equal to the approach velocity. Therefore, the unloading time in elasticplastic region can be calculated solely by using the elastic unloading equation, Eq. (12). Figure 4 illustrates the normalized elastic-plastic total loading time and the normalized unloading time against the normalized approach velocity. The total loading time also increases with increasing approach velocity.

The normalized contact duration and the normalized indentation depth in the elastic-plastic region are displayed in Fig. 5. This result indicates that either the contact duration or indentation depth has a moderate rising

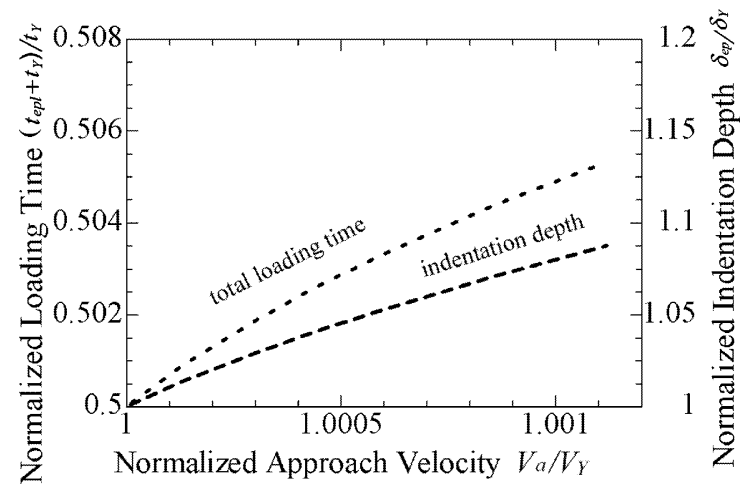

Fig. 3 Normalized loading time and normalized indentation depth as a function of normalized approach velocity of circular plate for elastic-plastic loading and unloading level in the elastic-plastic region.

\section{Plastic Collision}

\section{1 Plastic loading}

The plastic deformation begins when the mean contact pressure, $P_{P Y}$, equals $2.4 Y^{(4)}$. When plastic deformation begins the impact velocity is the approach velocity, $V_{P Y}$, and the maximum indention is $\delta_{P Y}$.

It is suggested that the total plastic loading time, $t_{\text {pltotal }}$, is the sum of four parts: the period of plastic loading, $t_{p l}$, the period of elastic-plastic loading, $t_{e p l}$, the period of elastic loading, $t_{Y}$, and the period of elastic unloading, $t_{\text {punl }}$.

$$
t_{\text {pltotal }}=t_{Y}+t_{\text {epl }}+t_{p l}+t_{\text {punl }}
$$

The relationship between the interaction force and the normal indentation in fully plastic collision was given by Stronge ${ }^{(4)}$,

$$
\frac{P}{P_{Y}}=1.6\left[1+\frac{1}{4}\left(\frac{\pi E_{*}}{6 Y}\right)^{2} \frac{\delta_{Y}}{R}\left(\frac{\delta}{\delta_{Y}}-1\right)\right]^{1 / 2}
$$

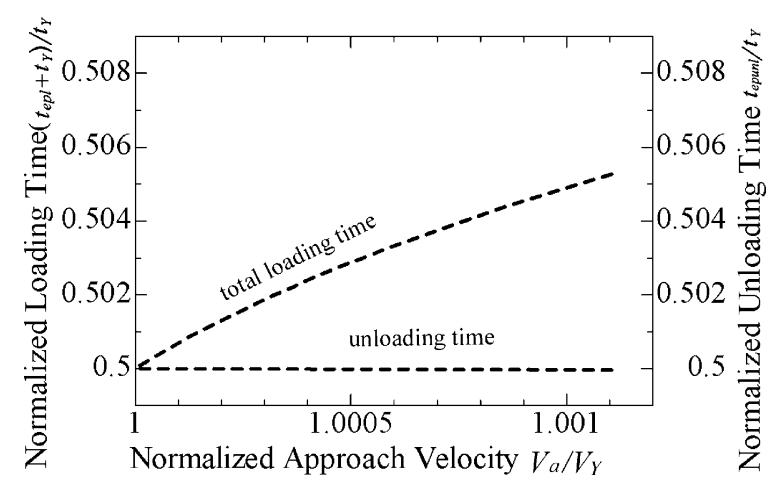

Fig. 4 Normalized total loading time and unloading time as a function of normalized approach velocity of circular plate

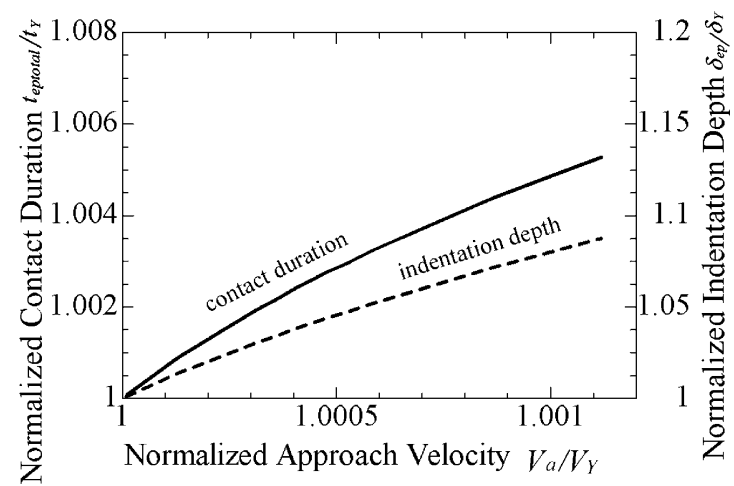

Fig. 5 Normalized contact duration and normalized indentation depth as a function of normalized approach velocity of circular plate for elastic plastic loading and unloading 
Substituting Eqs. (13) and (15) into Eq. (23), we have

$$
P=\frac{57.6 Y^{2} R}{\pi E_{*}}\left[1+\frac{\pi^{2} E_{*}^{2}}{144 R Y^{2}}\left(\delta-\delta_{Y}\right)\right]^{1 / 2}
$$

Then we have

$$
\begin{aligned}
& \frac{1}{2} m V_{a}^{2}-\frac{1}{2} m(\dot{\delta})^{2} \\
& \quad=\int_{\delta_{P Y}}^{\delta} \frac{57.6 Y^{2} R}{\pi E_{*}}\left[1+\frac{\pi^{2} E_{*}^{2}}{144 R Y^{2}}\left(\delta-\delta_{Y}\right)\right]^{1 / 2} d \delta
\end{aligned}
$$

and

$$
\begin{gathered}
V_{a}^{2}-(\dot{\delta})^{2}=\frac{11059.2 Y^{4} R^{2}}{m \pi^{3} E_{*}^{3}}\left\{\left[1+\frac{\pi^{2} E_{*}^{2}\left(\delta-\delta_{Y}\right)}{144 R Y^{2}}\right]^{3 / 2}\right. \\
\left.-\left[1+\frac{\pi^{2} E_{*}^{2}\left(\delta_{P Y}-\delta_{Y}\right)}{144 R Y^{2}}\right]^{3 / 2}\right\}
\end{gathered}
$$

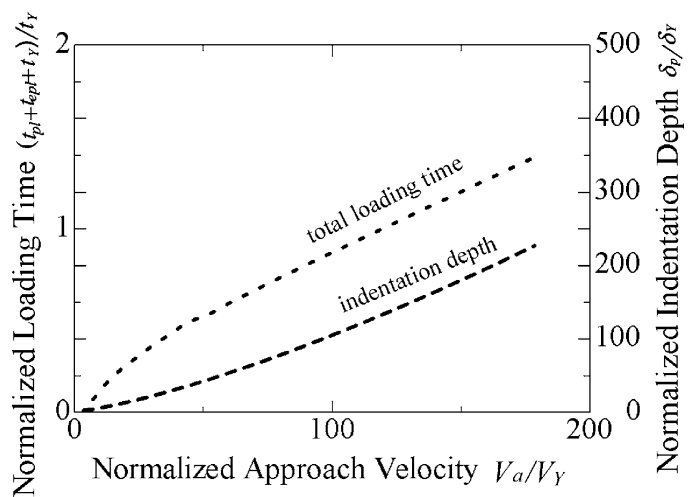

Fig. 6 Normalized loading time and normalized indentation depth as a function of normalized approach velocity of circular plate for plastic loading and unloading

Since the maximum indentation $\delta_{m}$ acts when the approach velocity is $V_{a}$, we have

$$
V_{a}^{2}=V_{P Y}^{2}+\frac{11059.2 Y^{4} R^{2}}{m \pi^{3} E_{*}^{3}}\left\{\left[1+\frac{\pi^{2} E_{*}^{2}\left(\delta_{m}-\delta_{Y}\right)}{144 R Y^{2}}\right]^{3 / 2}-\left[1+\frac{\pi^{2} E_{*}^{2}\left(\delta_{P Y}-\delta_{Y}\right)}{144 R Y^{2}}\right]^{3 / 2}\right\}
$$

Using Eqs. (30) and (31), the differential equation of the loading time for the plastic loading is given by

$$
t=\int_{\delta_{P Y} / \delta_{m}}^{1} \frac{\delta_{m} d x}{\sqrt{V_{P Y}^{2}+\frac{11059.2 Y^{4} R^{2}}{m \pi^{3} E_{*}^{3}}\left\{\left[1+\frac{\pi^{2} E_{*}^{2}\left(\delta_{m}-\delta_{Y}\right)}{144 R Y^{2}}\right]^{3 / 2}-\left[1+\frac{\pi^{2} E_{*}^{2}\left(\delta_{m} x-\delta_{Y}\right)}{144 R Y^{2}}\right]^{3 / 2}\right.}}
$$

where $\delta / \delta_{m}=x$.

Figure 6 illustrates the results of plastic loading time in Eq. (32). The normalized loading time and indentation depth increases with increasing the normalized approach velocity.

\subsection{COR and coefficient of indention}

The strain energy stored during compression drives the two bodies apart until they separate with some relative velocity. The COR is defined normally by the ratio of the approach velocity to the rebound velocity. However, the unloading time is dependent on the rebound velocity of circular plate in either elastic-plastic region or plastic region, which is obtained from COR of the collision.

For the elastic rebound process, Stronge ${ }^{(4)}$ has proposed two assumptions for the impact of an infinitely long cylinder. The first is that the maximum half width of loading contact surface, $b_{a}$, equals to the half width of unloading contact surface, $b_{r}$, that is, $b_{a}=b_{r}$. The second is that during elastic unloading the change in contact region are geometrically similar to the change during loading. During the elastic unloading the curvature changes in the contact region are geometrically similar to the changes that occur during the loading process. Thus

$$
\frac{\delta_{Y}}{R}=\frac{\delta_{r}}{\bar{R}} \leq \frac{\delta_{a}}{R}
$$

where $\bar{R}$ is an unloaded curvature ${ }^{(4)}$ that relates to the contact area. It does not equal to the initial value of the effective curvature, $R$. The unloading indentation, $\delta_{r}$, during the unloading process is less than the loading indentation, $\delta_{a}$. However, the ratio of unloading indentation to curvature would not always be maintained constant for any collision velocity as shown in Eq. (33).

Unlike infinitely long cylinders, the shape of indentation by the collision of circular plate is more complicated particularly around its edges. In order to take account of the actual thickness of circular plate or the length of cylinder, a new coefficient of indentation (COI), $\xi$, is proposed here as the following equation.

$$
\xi \frac{\delta_{Y}}{R}=\frac{\delta_{r}}{\bar{R}} \leq \frac{\delta_{a}}{R}
$$

According to Eqs. (1), (4) and (8) with the assumption of Eq. (34), the unloading indentation, the force and rebound velocity are expressed by 


$$
\begin{aligned}
& \delta_{r}=\frac{b_{r}^{2}}{\bar{R}}\left[\ln \left(\frac{4 \bar{R}}{b_{r}}\right)-\frac{1}{2}\right]=\frac{2 P_{r}}{\pi E_{*}}\left[\ln \left(\frac{4 R \delta_{r} \pi E_{*}}{\xi \delta_{Y} P_{r}}\right)-1\right] \\
& V_{r}^{2}=\frac{2 P_{r}^{2}}{m E_{*}}\left[\ln \left(\frac{4 R E_{*}}{P_{r}}\right)-\frac{3}{2}\right]
\end{aligned}
$$

The ratio of the rebound velocity to the approach velocity is the COR (theoretical COR) that varies with varying the COI.

\subsection{COR and hybrid nonlinear collision model}

The linear viscoelastic model composed of a linear spring and a linear dashpot gives a COR that is independent of the normal impact speed. For many collision pairs, a COR that decreases with increasing relative velocity at incidence is required in order to represent experimental measurements. A nonlinear viscoelastic model is a means of achieving this velocity dependence. One possibility is a linear spring in parallel with a nonlinear dashpot.

For elastic spheres, Simon ${ }^{(10)}$ suggested a hybrid relation between the contact force $F$ and relative displacement $x$,

$$
F=-k|x|^{1 / 2}(x+c|x| \dot{x}), \quad \dot{x}(0) \equiv-v_{0}<0
$$

where the elastic stiffness $k$ can be obtained from Hertz relation $k=4 E_{*} R_{*}^{1 / 2} / 3^{(1)}$ and $c$ is a damping coefficient. Chatterjee $^{(11)}$ had identified a close approximation for COR as shown,

$$
e \approx\left[-c V_{a}+\exp \left(0.4 c V_{a}\right)\right]^{-1}
$$

For a collision of aluminum circular plate and a plate, Lu et al. ${ }^{(5)}$ has used the hybrid collision model for his propose of describing a collision of aluminum circular plate to planar surface. The COR is given,

$$
\begin{aligned}
& e \approx\left[F_{0}\left(V_{a}\right)+\exp \left(-0.4 \times F_{0}\left(V_{a}\right)\right)\right]^{-1} \\
& F_{0}\left(V_{a}\right)=0.16 V_{a}-0.1
\end{aligned}
$$

which has an excellent fit to his experimental data in Fig. 7. Figure 7 also illustrates that the COI, $\xi$, has an effect on the coefficient of restitution. If $\xi$ is properly chosen, the theoretical COR fit excellently to the hybrid COR.

Figures 7 and 8 represent the theoretical results of COR when $\xi$ takes values from 1 to 3 . In the region of

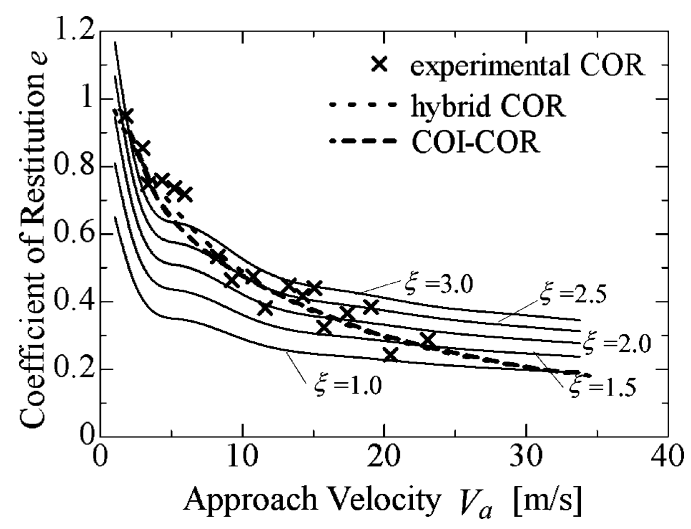

Fig. 7 The effect of the coefficient of indentation, $\xi$ relative lower approach velocity, the result of theoretical COR is less than that of experimental COR when $\xi$ takes 1. However, the results of theoretical COR best fit to that of experimental COR when $\xi$ takes 3. With the increasing of approach velocity, $\xi$ decreases to 1 . In the region of relative higher approach velocity $\left(V_{a}>24 \mathrm{~m} / \mathrm{s}\right)$, the results of theoretical COR best fit to that of experimental COR when $\xi$ takes 1 .

\section{4 Elastic unloading time in plastic collision}

The elastic unloading time can be obtained from Eq. (12) and the rebound velocity obtained from Eq. (36) is dependent on the COR. The normalized total loading time and unloading time that are calculated by using COR for cylinders respectively and hybrid COR for the plastic collision are shown in Fig. 9.

With increasing the normalized approach velocity, the normalized loading time increases and the normalized unloading time decreases on the contrary. The normalized loading time and normalized unloading time have different effects when the normalized approach velocity increases. The contact duration is the sum of the loading time and the unloading time but the loading time is dominant. As shown in Fig. 10, the contact duration of circular plate at the plastic collision increases with increasing approach ve-

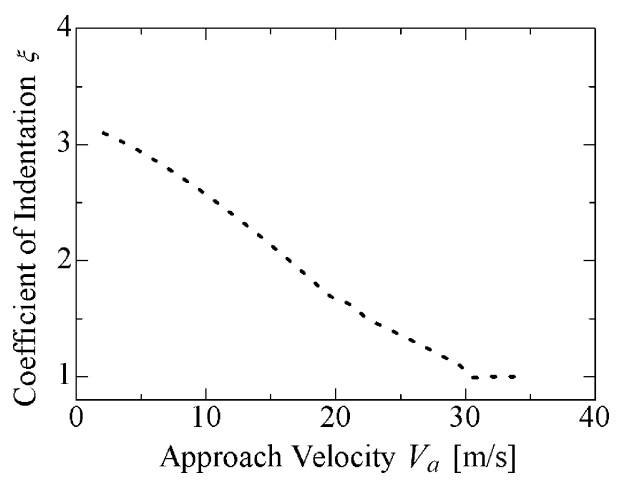

Fig. 8 The coefficient of indentation and approach velocity

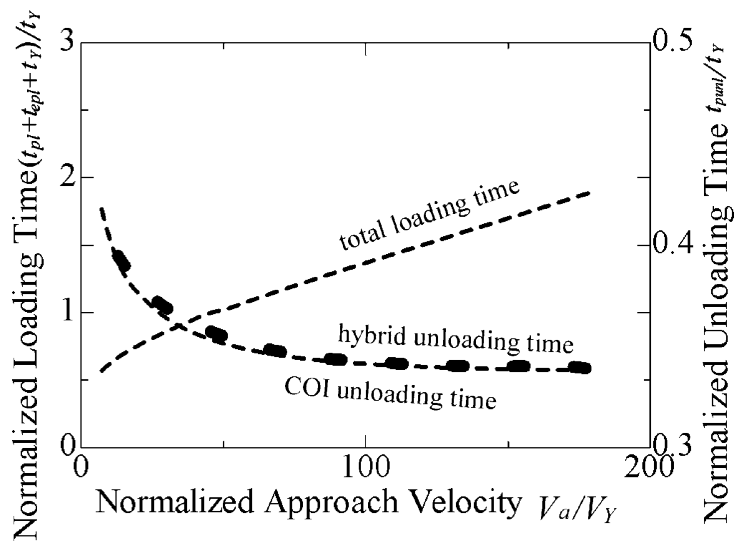

Fig. 9 Normalized total loading time and unloading time (hybrid and cylinder) as a function of normalized approach velocity of the plastic collision of circular plate 


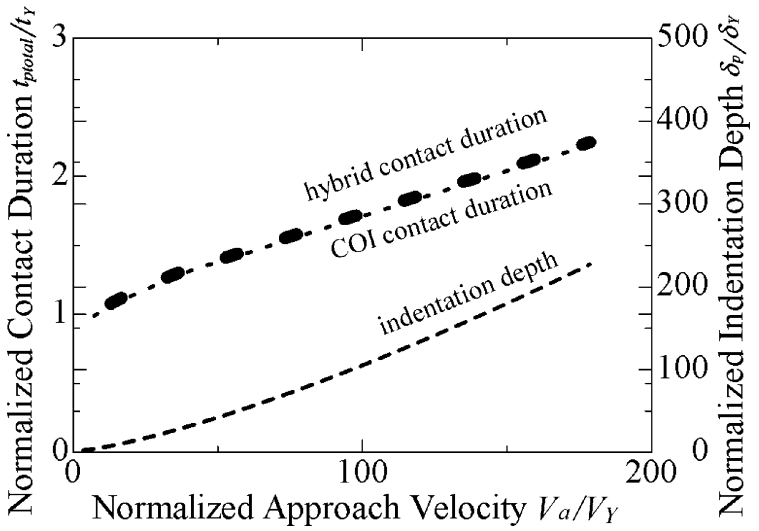

Fig. 10 The contact duration and normalized indentation depth as a function of normalized approach velocity of circular plate for plastic collision

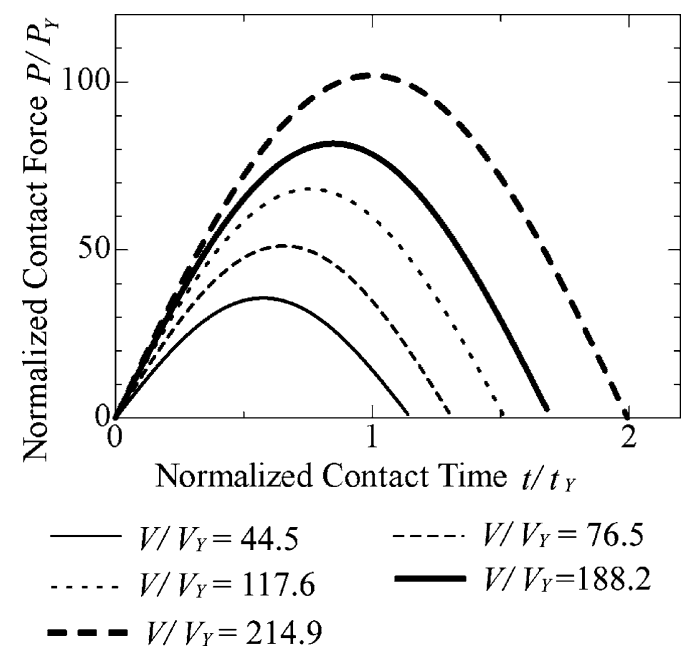

Fig. 11 The contact force history of the collision

locity.

\section{The Collision Pulse Shape}

According to the analysis in the section 4 , the contact duration can be theoretically calculated in the plastic loading and elastic unloading process. From Eqs. (28) and (35), the loading and unloading contact forces can be investigated corresponding to the loading normal indentation and the unloading normal indentation. Figure 11 shows the pulse shape about the collision of a circular plate of diameter $60 \mathrm{~mm}$ made of aluminum alloy (A6063) (Young's modulus, 69.7 GPa, Poisson's ratio, 0.33, yield stress, $50 \mathrm{MPa}$ ) to an aluminum alloy planar surface.

It is obvious that as the contact time becomes bigger, and the force also increases with increasing of the approach velocity. Also the force history of the collision process is expressed approximately by a half-sine wave and the width of the half-sine wave corresponds to the normalized contact duration in Fig. 10.

\section{Conclusion}

A theoretical method for investigating the contact duration and indentation depth in elastic and inelastic collision of circular plate and a planar surface is proposed. For obtaining the COR of collision, we introduce a hybrid nonlinear model method and COI method which takes account of the actual thickness of circular plate. The numerical results referring to the two methods offer an excellent fit to the experimental results of COR respectively.

Using the theoretical method, we may also predict the collision pulse shape can be expressed approximately by a half-sine wave and the force and contact duration may be calculated numerically.

\section{References}

(1) Timoshenko, S. and Goodier, J.N., Theory of Elasticity, 3rd Edn, (1951), pp.409-422, McGraw-Hill, New York, London et al.

( 2 ) Falon, E., Laroche, C. and Coste, C., Behavior of One Inelastic Ball Bouncing Repeatedly off the Ground, Eur. Phys. J., B, Vol.3 (1998), pp.45-57.

( 3 ) Landau, L.D. and Lifshitz, E.M., Theory of Elasticity, 3rd, (1986), pp.27-31, Pergamon Press.

( 4 ) Stronge, W.J., Impact Mechanics, (2000), pp.86-145, Cambridge University Press.

( 5 ) Lu, Z., Quan, C., Tanaka, K. and Nishida, M., Impact and Restitution of Rotating Circular Plate, The 54th Term General Meeting and Lecture Meeting, TokaiBranch, JSME, (in Japanese), (2005), pp.7-8.

(6) Gerl, F. and Zippelius, A., Coefficient of Restitution for Elastic Disks, Phys. Rev., E, Vol.59, No.2 (1999), pp.2361-2372.

( 7 ) Avanzini, F. and Davide, R., Modeling Collision Sounds: Non-Linear Contact Force, Proceedings of the COST G-6 Conference on Digital Audio Effects (DAFX-01), Limerick, Ireland, December 6-8, (2001).

( 8 ) Zhang, X. and Vu-Quoc, L., Modeling the Dependence of the Coefficient of Restitution on the Impact Velocity in Elasto-Plastic Collision, Int. J. Impact Engineering, Vol.27 (2002), pp.317-340.

(9) Johnson, K.L., Contact Mechanics, (1985), pp.129134, Cambridge University Press, Cambridge.

(10) Simon, R., Development of a Mathematical Tool for Evaluating Golf Club Performance, ASME Design Engineering Congress, New York, (1967).

(11) Chatterjee, A., Rigid Body Collision: Some General Considerations, New Collision Laws and Some Experimental Data, Ph.D. Dissertation, Cornell Univ., New York, (1997). 